\title{
Neuromorphic dynamics with optically injected quantum dot lasers
}

\author{
M. Dillane, ${ }^{1,2}$ J Robertson ${ }^{3}$ M. Peters,${ }^{1}$ A Hurtado, ${ }^{3}$ and B. Kelleher ${ }^{1,2}$ \\ ${ }^{1}$ Department of Physics, University College Cork, Ireland \\ ${ }^{2}$ Tyndall National Institute, University College Cork, Lee Maltings, Dyke Parade, Cork, Ireland \\ ${ }^{3}$ Institute of Photonics, SUPA Department of Physics, University of Strathclyde, \\ TIC Centre, 99 George Street, Glasgow G1 1RD, UK
}

(Dated: June 1, 2019)

\begin{abstract}
Optically injected quantum dot lasers display many unique nonlinear phenomena and are in particular, excellent testbeds for different forms of excitability. We analyse the recent discovery of Type II excitability in such devices. An optothermal instability leads to the phenomenon and while an underlying Hopf bifurcation is ultimately responsible for the observation, intriguingly there are two potential routes: One via a subcritical bifurcation and an associated bistable region and the other via a supercritical bifurcation and an associated canard explosion.
\end{abstract}

\section{INTRODUCTION}

The well-known limitations of conventional, digital computing for certain problems has led to an enormous interest in artificial neural networks. Neuromorphic photonics has emerged as one of the leading candidates for emulating the computational abilities of the brain with semiconductor lasers in particular leading the way given their compact size and ultrafast processing speeds and most importantly for this work, their ability to produce excitable responses in a variety of configurations [1] . Excitability of course was originally discovered in biological systems [2] but has been observed in a multitude of different systems including chemical, electrical and optical systems [3-11]. Small perturbations of the rest state lead to short phase space excursions. However, there exists a perturbation threshold above which the system undergoes a large phase space excursion corresponding to a pulse (or a spike as they are often called in neuronal studies). Following a pulse, there is a refractory time during which another excitation cannot be triggered [12].

Many photonic devices and device configurations have been shown to exhibit excitability and related phenomena. The optical feedback configuration provided one of the earliest examples of the phenomenon and continues to be a rich source of excitability $[6,9,10,13,14]$. Two section lasers employing saturable absorbers provide many examples with several novel laser types [1521]. Several schemes make use of lasers with two inherent lasing states including semiconductor ring lasers $[22,23]$, VCSELs [24] and dual state quantum dot (QD) lasers [26, 27]. Other authors have considered systems where lasers were not central to the excitable dynamic, such as $[28,29]$ where photonic crystal cavities were considered and $[30,31]$ where resonant tunnel photodiodes were used in conjunction with a laser. Central to the work presented here is the optical injection configuration which has provided many examples of single and multipulse excitability $[7,8,11,12,22,24,32-37]$. We note that these lists are non-exhaustive and we do not even consider approaches to neuromorphic photonics outside excitability such as reservoir computing [38]. See [1] for a recent comprehensive review of neuromorphic photonics.

Depending on the system specifics, one typically obtains either Type I excitability or Type II excitability [39], but not both. Recently however, we have demonstrated that optically injected QD lasers can display both Type I and Type II excitability [40]. We investigate the emergence of the Type II phenomenon in greater depth in this work. We first review some of the known results for excitability with optically injected QD lasers.

\section{EXCITABILITY IN OPTICALLY INJECTED QD LASERS}

It has been shown in several previous works that Type I excitability is generic in optically injected edge emitting semiconductor lasers for weak injection levels [7, 11, 41]. In particular, it arises with injected QD lasers, and because of the high relaxation oscillation damping of these devices it arises in many unique forms. At extremely low injection levels, QD lasers behave just like idealised Adler phase oscillators [34]. As the injection level is increased the details of the excitable dynamics evolves. $2 \pi$ phase rotations are obtained for both positive and negative detuning at low injection levels [33] and these phase slips can be deterministically triggered as shown in [36]. For higher injection levels QD lasers display bursting with groups of pulses rather than isolated pulses emitted [34]. Uniquely among semiconductor lasers, QD devices can emit from multiple energy states and this too can lead to novel dual excitable regimes such as non-Adler Type I excitability [26] and tunable bursting regimes [27] featuring slow passage through a Hopf bifurcation. The mutual coupling configuration is the natural extension of optical injection system and when mutually coupled with delay, excitable pulse trains are generated by QD lasers [42] which could be of interest for networks of artificial neurons.

Type II excitability is less well known in the optically injected laser system. It typically manifests in fast-slow systems and the Van der Pol Fitzhugh Nagumo model is the prototypical model used to describe it. Recently, such excitability has been reported for optically injected 
QD devices: When a QD laser is optically injected with high injection strength, excitable square pulses are obtained [40]. The duration of each square is constant and on the order of a microsecond but the rise and fall times of each square are on the order of a nanosecond. Physically, a bistability is broken by an optothermal coupling, leading to a deterministic evolution of the detuning between the master and slave lasers. This manifests as a deterministic square wave cycle between the remnants of the two states. Close to this deterministic cycle, the system is excitable and noise induced pulsations are obtained as shown in [40]. Such optothermal coupling has also previously been described in [27, 29, 43-47]. In [47] the authors also identified a canard explosion using an injected semiconductor optical amplifier.

In this work we show that the pulses can be triggered deterministically with a well defined threshold above which pulses can be triggered with $100 \%$ efficiency. We analyse the phase excursion of the trajectory and find that it is a bounded cycle. Such cycles are often (but not always) associated with Hopf bifurcations [48, 49] and so one might expect to find such a bifurcation. The conventional route to Type II excitability is via a subcritical Hopf bifurcation. The rest state is bistable with a large amplitude cycle close to the excitable regime and after passing the bifurcation, the large amplitude cycle is the only attractor in the system. There is another alternative to this scenario which is to find a supercritical Hopf bifurcation. The large amplitude excitable trajectories then arise due to a canard explosion. In this case, the small amplitude oscillations born in the bifurcation quickly lead to a large amplitude cycle. Intriguingly, we find both routes numerically. Which one arises depends on the ratio of the photon and carrier lifetimes.

\section{EXPERIMENT}

A $300 \mu \mathrm{m}$ long InAs based QD laser similar to the one used in [40] was optically injected with a tunable laser source (TLS) in a unidirectional master-slave configuration. The TLS had a linewidth of approximately $100 \mathrm{kHz}$. A polarization controller was used to maximise coupling between the master and slave. The pump laser was operated at $40 \mathrm{~mA}, 1.25$ times threshold. FIG.1 shows the evolution of the intensity with detuning (as previously shown in [40] but displayed again here for completeness).We begin with large negative detuning, where the frequency of the master is far less than that of the slave. Here a simple limit cycle was obtained. By increasing the detuning the QD laser became phase locked to the master. Increasing in detuning again, isolated square pulses induced by noise began to appear, as seen in FIG 2 (a), where the low intensity section corresponds to the phase locked state. As the detuning is further increased, square pulses with random interpulse times become more frequent, FIG. 1 (a). Increasing the detuning again, the noise induced train gives way to a periodic square wave train FIG. 1 (b). Continuing to increase the detuning, the deterministic train gives way to noise induced square dropouts FIG. 1 (c) and finally the slave becomes phase locked again, but to the high intensity section.
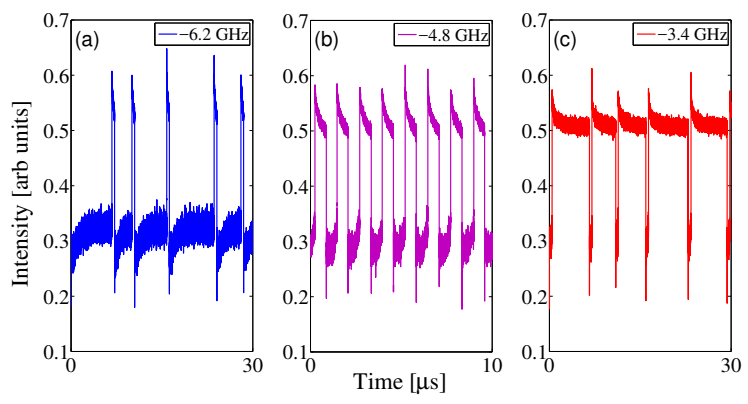

FIG. 1: Experimental data where (a) shows noise induced pulse train, (b) shows periodic square pulse train and (c) shows noise induced square dropouts.

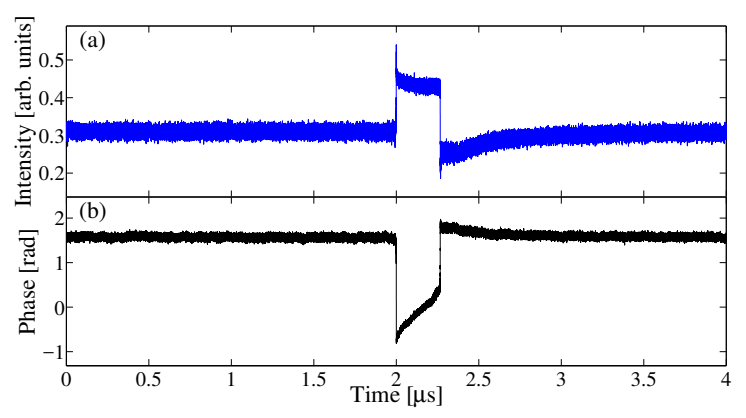

FIG. 2: (a) Timetrace of a noise induced excitable pulse at approximately $-6.6 \mathrm{GHz}$ detuning. (b) The phase associated with the timetrace from (a). The phase undergoes a change of $2.4 \mathrm{rad}$ at the onset of a square pulse and over the duration of the pulse the phase undergoes a slow change of approximately $1.4 \mathrm{rad}$.

While the intensity behaviour has been reported in [40], the underlying phase trajectories have heretofore not been discussed or studied. We investigate the phase dynamics of the squares in this work, using the phase resolving technique developed in [50]. This method uses a $3 \times 3$ coupler. One input arm receives light from the slave, another receives $10 \%$ from the TLS and the third is left empty. All three outputs are detected using $12 \mathrm{GHz}$ detectors connected to a real time oscilloscope. FIG. 2 shows a noise induced excitable square pulse and its phase. Over the duration of the high intensity section of the square pulse, the phase undergoes a slow change of approximately $1.4 \mathrm{rad}$. This slow change can also be seen in the the long narrow section of the $2 \mathrm{D}$ phase diagram in FIG. 3. The more concentrated shape around $(0,0.4)$ corresponds to the approximately constant, low intensity section in FIG 2 (a). The phase of lower intensity section also remains approximately constant, FIG. 2 (b). The phase trajectory is clearly bounded. The slow phase 


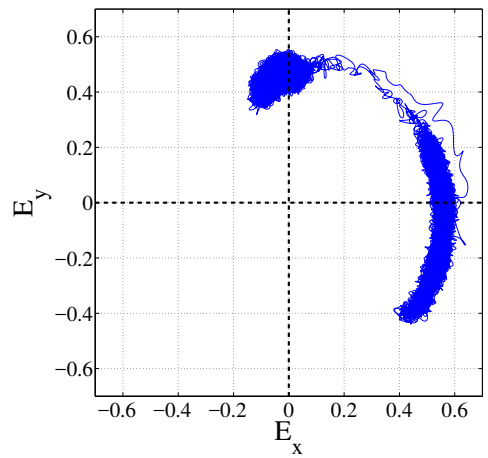

FIG. 3: Phasor diagram for the slave laser field. Corresponding to the pulse seen in FIG 2.

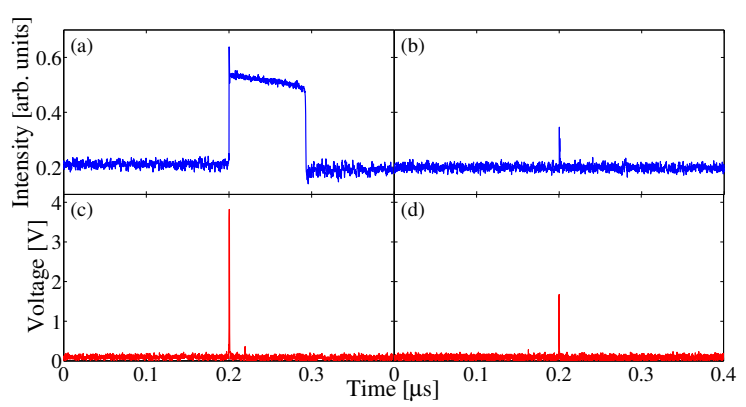

FIG. 4: (a) and (b) show the output from the slave laser. (c) and (d) show the electric pulses of different amplitudes that were sent to the phase modulator in order to perturb the injected light. (a) is a successfully triggered optical square pulse and was triggered by an electrical pulse (c) with an amplitude of $3.82 \mathrm{~V}$. The smaller perturbation (d) with an amplitude of $1.67 \mathrm{~V}$,

fails to excite a square pulse as can be seen in (b)

evolution through the square corresponds physically to the slow evolution of the effective detuning arising from the optothermal coupling [40].

While the presence of stochastic pulses allow us to conclude on the excitable nature of the system, for applications one would typically need to deterministically trigger pulses $[35,36]$. To investigate this we use a $\mathrm{LiNbO}_{3}$ phase modulator to perturb the injected light. The modulator is connected to a pulse generator that generates pulses with a full width half maximum of approximately $1 \mathrm{~ns}$ and a rise time of $100 \mathrm{ps}$. By varying the perturbation amplitude the presence or otherwise of a threshold can be investigated. FIG. 4 shows two perturbations and the resulting laser responses for the phase locked slave laser biased at $36.5 \mathrm{~mA}$ (1.14 times threshold). In FIG. 4 (c) a large amplitude voltage spike is applied and a square pulse (FIG. 4 (a)) is excited in the slave laser. In FIG. 4 (d) a low amplitude voltage pulse is applied and a square is not excited in the laser. Instead, the intensity returns to the steady state via a short phase space trajectory (FIG. 4 (b)). The probability of excitation of a

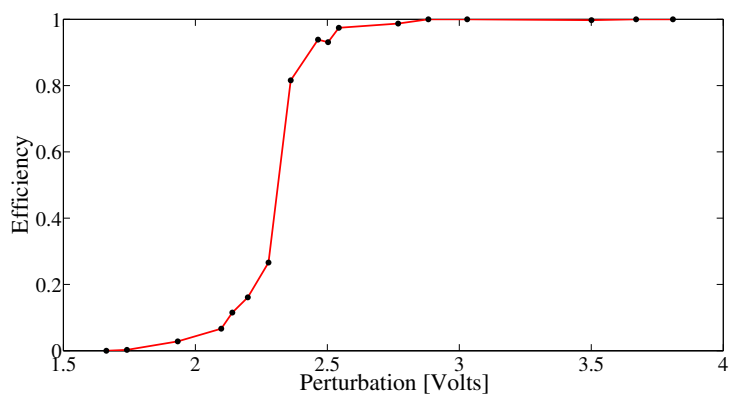

FIG. 5: Efficiency curve for deterministically triggered square pulses. There is a threshold at approximately $3 \mathrm{~V}$. If the strength of the perturbation is greater that this than there is a a high probability that a pulse would be fired.

pulse for a particular perturbation strength is calculated by finding the number of successful pulses triggered after making 391 consecutive perturbations. An efficiency curve can then be generated as shown in FIG. 5. There is a clear threshold showing that the square pulses can be deterministically triggered with a sufficiently strong voltage pulse, clearly demonstrating the excitable nature of the square pulses.

To better understand the phenomenon we undertake a numerical analysis of the system by employing rate equation models of the system. As shown in $[51,52]$ the high relaxation oscillation damping of QD lasers means that many of the phenomena observed with injected QD devices can be understood via an injected Class A laser model. Such an approximation was used in [40] and we use it again as our first model in this work.

\section{CLASS A LASER MODEL}

Our first numerical analysis uses the rate equations for an optically injected Class A laser [51-54], supplemented by an extra equation describing the optothermal coupling. The model is

$$
\begin{aligned}
\dot{R} & =\frac{P-R^{2}}{1+2 R^{2}} R+K \cos \phi \\
\dot{\phi} & =\alpha \frac{P-R^{2}}{1+2 R^{2}}-(\Delta-\omega)-\frac{K}{R} \sin \phi \\
\dot{\omega} & =-\gamma\left(\omega-c R^{2}\right),
\end{aligned}
$$

where $R$ is the slave field amplitude, $K$ measures the injection strength, $\phi$ is the phase of the slave minus that of the master, $\Delta$ is the nominal angular detuning, $\alpha$ is the phase amplitude coupling factor and $P$ is the pumping current above threshold. $\omega$ is the thermal change in the detuning, $\gamma$ gives the slow characteristic time scale for the thermal effects, and $c$ is the coupling between the thermal dynamics and the intensity. Time is expressed in units 
of the photon lifetime $\tau_{p h}$. While discussing the Class A model the parameters are fixed as follows: $\alpha=2, K=$ $0.7104, P=0.5, c=0.1, \gamma=5 \times 10^{-6}$. (We note that the results are robust to changes in $\alpha$ and $\gamma$. For example, $\gamma$ can be changed by several orders of magnitude while retaining the qualitative features analysed here.)

For high injection strengths and in the absence of thermal effects, a phase-locked bistability is predicted $[51,52]$. When thermal effects are included, a square wave regime is found [40]. Physically one can reason that the optothermal coupling breaks this underlying bistability and that the square trajectory results from a deterministic detuning sweep along the underlying steady states. To examine this, we first plot the bifurcation diagram for the bare system in the bistable region, the blue curve in FIG. 6 (found using the continuation package AUTO). The stable steady states arise from saddle-node bifurcations and clearly overlap forming the bistable region. We then include the optothermal coupling and plot the intensity of the square wave regime over the bifurcation curve (red). The findings justify our physical intuition. The squares result from the slow deterministic evolution of the detuning. The intensity of the square corresponds to the steady state intensity of the bare system. The bistability (blue) is destroyed by an optothermal coupling and is replaced by a deterministic cycle represented by the red curve.

A natural question is what bifurcation leads to this square wave regime. Via a combination of direct simulation and the numerical continuation software package AUTO we find a new Hopf bifurcation in the system introduced via the thermal coupling which destabilises the phase locked state and breaks the bistability. FIG.7 is a bifurcation diagram showing the location of this bifurcation $\Delta_{H}$. Directly after the bifurcation there is a small amplitude cycle as shown in FIG.7 inset (a). However, after a very short range of detuning, large amplitude cycles appear, FIG.7 inset (b). This occurs via a canard explosion.

Close to the bifurcation the system is excitable. By including noise in the simulations one can obtain noise induced excitable events as demonstrated in [40]. We complement this analysis here by taking a noise free system but investigate excitability by applying discrete perturbations which can lead to trajectories around the large amplitude cycle for a sufficiently large perturbation. One can obtain such an excitable trajectory at both sides of the bifurcation point $\Delta_{H}$, i.e. when the system is either in the regime of constant intensity output or in the small amplitude oscillation regime, shown in FIG.7. The response of the system depends on the amplitude of the perturbation. FIG.8 shows two responses to slightly different phase perturbations, when operating in the constant intensity regime, where $\Delta<\Delta_{H}$. One response simply yields a short phase space trajectory back to the stable state (black), while the bigger perturbation is just above the excitable threshold and yields an excitable pulse (red).

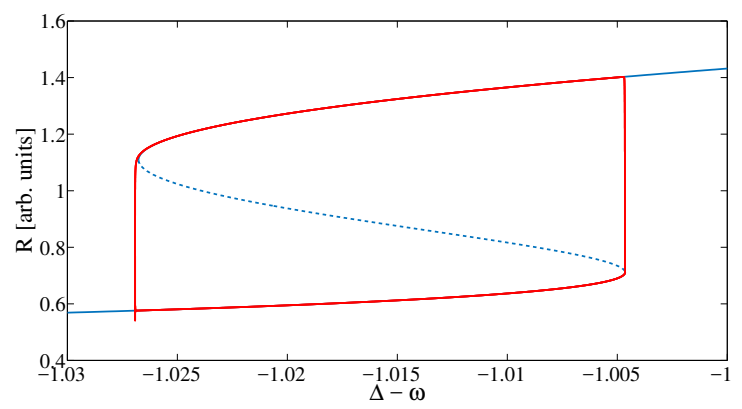

FIG. 6: Bifurcation diagram using the Class A model. The blue curve shows the saddle node structure of the bare system (no optothermal coupling, $\mathrm{c}=0$ ). The solid lines represent stable solutions and the dashed lines represent unstable solutions. There is a clear bistability.

The red curve shows the trajectory of FIG. 7(b) obtained at at $\Delta=-0.9540124775$ with a finite optothermal coupling, $c=0.1$ and $\gamma=5 \times 10^{-6}$. For both cases $K=0.7104$.

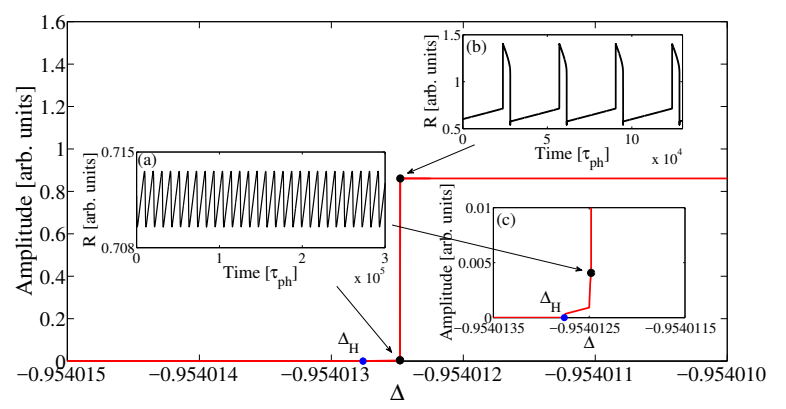

FIG. 7: (Colour online) Bifurcation diagram showing the new Hopf bifurcation created by the optothermal coupling. The Hopf point $\Delta_{H}=-0.95401276$ is shown with a blue dot. Examples of small and large amplitude cycles at $\Delta=-0.95401248$ and $\Delta=-0.9540124775$ are shown in insets (a) and (b), where their amplitudes are approximately 0.004 and 0.86 respectively. Zero corresponds to constant output. Inset (c) zooms in on the canard explosion and the associated region of small amplitude oscillations. $K=0.7104$ throughout.

We recognise that the model is overly simple and that it would be preferable - and indeed, crucial - to reproduce the results using a model explicitly designed for QD lasers and so we turn to this below.

\section{QUANTUM DOT LASER MODEL}

In order to best compare with existing work we use the rate equation model of $[51,55]$. This consists of equations for the electric field amplitude $R$ of the laser, the phase $\phi$ in the frame of the master and the carrier density in the wetting layer $n$. The occupation probability of the 


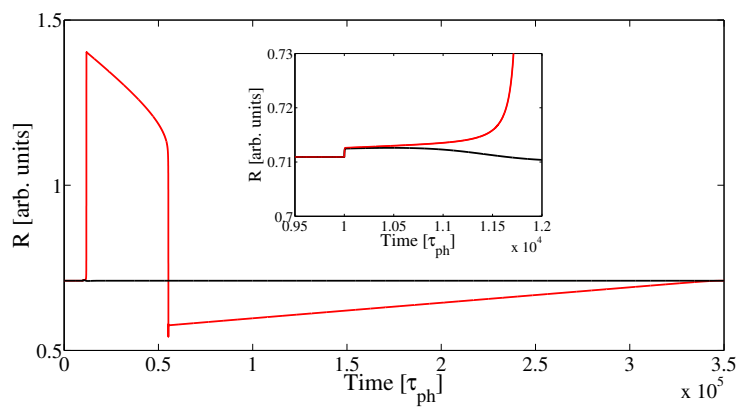

FIG. 8: (Color online) Two examples of perturbed trajectories. In the black trace a perturbation of $\delta \phi=0.0011$ was applied at $t=1000$ yielding a short trajectory back to the steady state behaviour. In the red trace a perturbation of $\delta \phi=0.0012$ was applied yielding an excitable trajectory. $K=0.7104$, $\Delta=-0.9541$. The inset zooms in on the perturbation.

dots has been adiabatically eliminated as in [51]. (The adiabatic elimination arises from the small parameter $\varepsilon$ which gives the deviation of the dimensionless differential gain from 1. See [51] and references therein for further details.) Again, we supplement the model with an extra equation describing the optothermal coupling, just as in the Class A model. The equations are as follows.

$$
\begin{aligned}
\dot{R} & =1-\frac{2\left(1+R^{2}\right)}{2 B \varepsilon n} R+\frac{K}{\varepsilon} \cos (\phi) \\
\dot{\phi} & =-\frac{\Delta-\omega}{\varepsilon}+1-\frac{2\left(1+R^{2}\right)}{2 B \varepsilon n} \alpha-\frac{K}{\varepsilon R} \sin (\phi)+D \xi(T) \\
\dot{n} & =\frac{1}{\varepsilon} \eta\left(J-n-2\left(1+R^{2}\right)\right) \\
\dot{\omega} & =-\frac{\gamma}{\varepsilon}\left(\omega-c R^{2}\right)
\end{aligned}
$$

Here, the dot means differentiation with respect to $T=\varepsilon t / \tau_{p h} . \quad J$ is the pump current per dot and $\alpha$ is the linewidth enhancement factor. $B \equiv \tau \tau_{c a p}^{-1}$ and $\eta \equiv \tau_{p h} \tau^{-1}$ where $\tau$ and $\tau_{c a p}$ denote the carrier recombination and capture times, respectively. $J$ represents the pump current per dot. $D \xi(T)$ is a white noise term, where $\xi(T)$ is a Wiener process and $D$ is the magnitude. To allow direct comparison with FIG 1.14 in [51] we use the same parameter values and keep them fixed: $\alpha=1.2$, $J=5.14$ (1.5 times threshold), $B=70, \varepsilon=0.02$ and $\eta=2 \times 10^{-3}$. The optothermal effect is introduced in a similar way to how it was included in the Class A model, $\gamma$ again provides the slow thermal relaxation time and $c$ is the optothermal coupling constant. We fix $c=0.03$ and $\gamma=5 \times 10^{-5}$. Our control parameters are the injection rate $K$ and the detuning $\Delta$ defined as the frequency of the master laser minus that of the slave laser.

The bare system with no optothermal coupling yields a region of phase-locked bistability [51]. Including the optothermal coupling we find as in section IV that a square
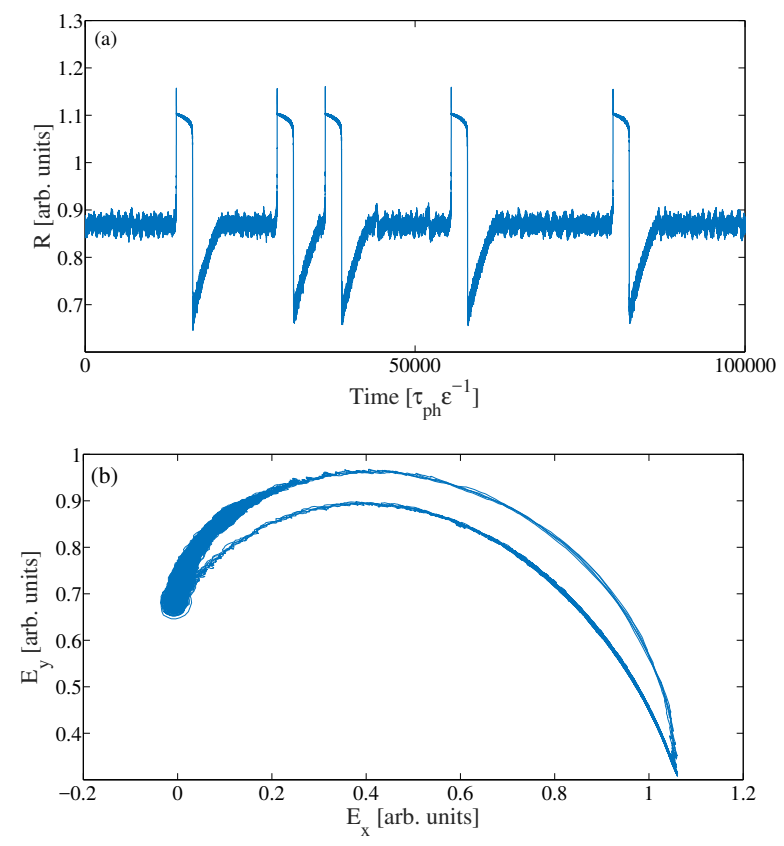

FIG. 9: (a) Noise induced excitable squares pulses found for $K=0.04, \Delta=-0.029525, D=0.01$, $\gamma=5 \times 10^{-6}$ and $\eta=2 \times 10^{-3}$. (b) shows the associated phasor trajctory.

wave regime can be obtained, see FIG. 9 (a). Again, direct simulation demonstrates that the system is excitable close to this region with pulses arising from discrete perturbations and via noise, $D=0.01$. FIG. 9 (a) shows a noise induced pulse in the excitable region and FIG. 9 (b) shows the associated phasor trajectory. The agreement with the experiment is excellent. Admittedly, the overall phase rotation is smaller than that found experimentally but we ascribe this to the relatively low value of $\alpha$ used (deliberately to allow comparison with the existing results in $[51,55]$.

As with the Class A model, we again find that the square wave trajectory follows the bistable steady states of the underlying bare system as shown in FIG. 10. As for the bifurcation responsible for the appearance of the squares, we again find a Hopf bifurcation at their onset. However, unlike the Class A case, we find a subcritical Hopf bifurcation. The system is in fact bistable between the phase-locked solution and a square wave train just before the Hopf is reached. FIG.11 shows the two stable states for the same control parameters but for a noise free system, $D=0$. Only the initial conditions differ. Thus, the excitability arises via the conventional, subcritical Type II dynamic for the QD model with these parameters. However, as we show now, we can regain the canard explosion feature by changing just one parameter.

The Class A model is the limit of the more conventional Class B model as the ratio of the photon lifetime to the carrier lifetime tends to infinity ( $\eta$ in our QD model). However, as shown in [52] many features of the Class 


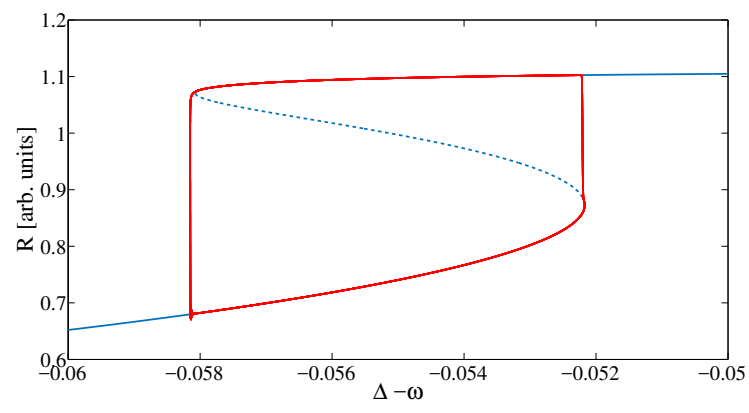

FIG. 10: Bifurcation diagram using the QD model. The blue curve shows the saddle node structure of the bare system (no optothermal coupling, $\mathrm{c}=0$ ). The solid lines represent stable solutions and the dashed lines represent unstable solutions. There is a clear bistability. The red

curve shows the trajectory shown in FIG. 12(b) at

$\Delta=-0.02931155$ for finite optothermal coupling, $c=0.03$ and $\gamma=5 \times 10^{-6}$. For both cases $K=0.04$,

$$
D=0 \text { and } \eta=2 \times 10^{-3} \text {. }
$$

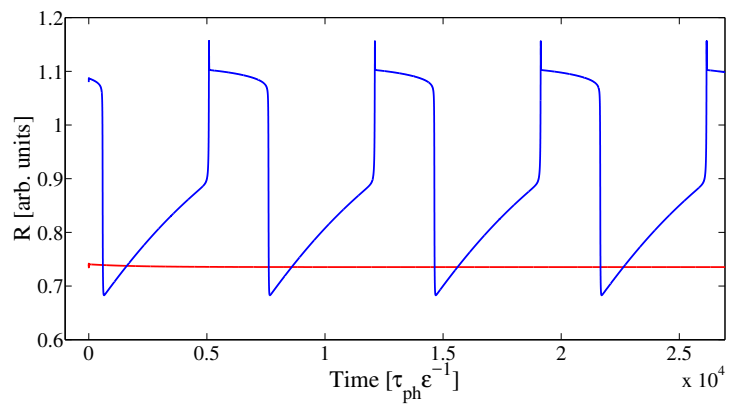

FIG. 11: After passing the subcritical Hopf bifurcation point a bistability is creating and the two solutions are

shown above. The solution the system evolves into depends on the initial conditions. Initial conditions for the constant trace are $R=0.735, \phi=1.560, n=2.06$

and $\omega=0.016$. For the square trace the initial conditions are $R=1.08, \phi=0.667, n=0.795$ and $\omega=0.028$. The operating parameters are the same for both $K=0.04, \Delta=-0.02929, \eta=2 \times 10^{-3}$, $\gamma=5 \times 10^{-6}$ and $D=0$.

A system arise also in the standard Class B system for finite ratio. This is also the case here. We find that for very modest $\eta$ the Hopf bifurcation becomes supercritical and we recover the canard explosion. FIG.12 shows this clearly, where $\eta=0.03$.

\section{CONCLUSION}

The question now arises as to which version is observed in the experiment. The traces for the noise induced pulses are extremely similar both for the subcritical and supercritical Hopf cases and both match the experiment

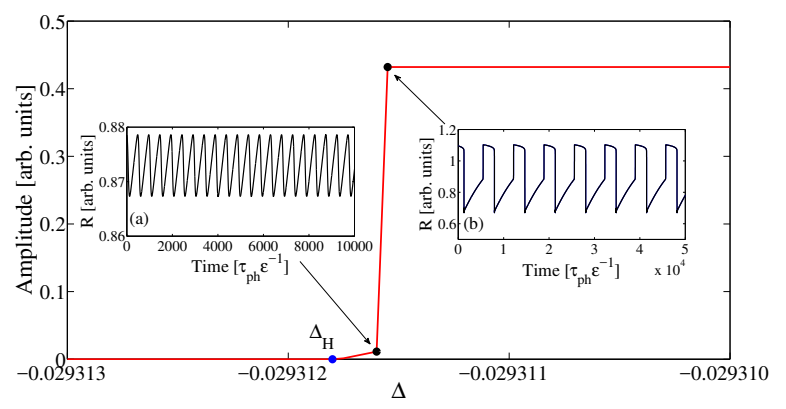

FIG. 12: Bifurcation diagram for the supercritical Hopf case showing the amplitude of the cycle versus the detuning. (Thus, zero corresponds to constant output.) Small amplitude oscillations are obtained just after the Hopf point (blue dot) $\Delta_{H}=-0.0293118$. A canard explosion then leads to the creation of a large amplitude cycle. The insets show two characteristic examples of the output. $K=0.04, D=0, \eta=0.03, c=0.03$ and $\gamma=5 \times 10^{-6}$ throughout. The insets show $\Delta=-0.0293116$ and $\Delta=-0.02931155$ for the small amplitude and large amplitude cycles with amplitudes of 0.01 and 0.43 respectively.

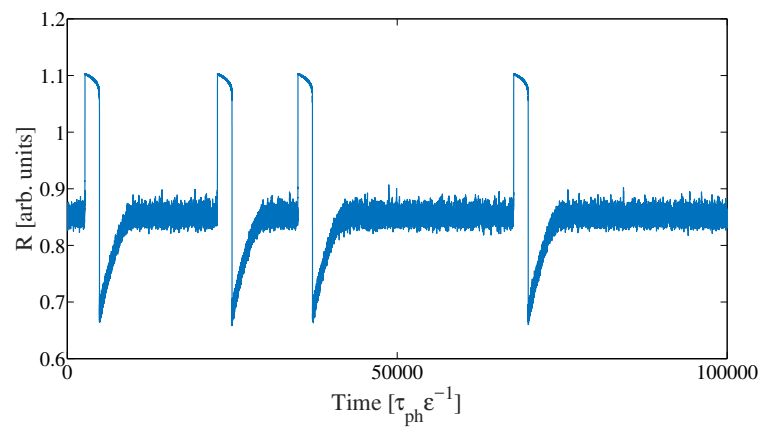

FIG. 13: Noise induced square pulses for the supercritical Hopf case. $K=0.04, \Delta=-0.0304$, $\gamma=5 \times 10^{-6}, \eta=0.03, D=0.01$.

well. One feature does stand out: The overshoot at the beginning of each square in the experimental traces. This is reproduced by the QD model with the subcritical Hopf parameters but not with the Class A and not with the supercritical Hopf QD case. We ascribe this overshoot to the lower relaxation oscillation damping of the subcritcal case compared with both other cases. It is thus tempting to identify the subcritical case as the underlying scenario. Further, the material parameters for the subcritical case agree with those in the literature while the supercritical case requires a much higher photon lifetime/carrier lifetime ratio. Of course, a more thorough bifurcation analysis is warranted both experimentally and numerically. It would be very interesting to analyse experimentally a device identical in all respects apart from the photon lifetime (via reflective coatings for example) to see if the supercritical case and the associated canard explosion could 
be identified. Perhaps even by pumping the device only slightly above threshold it would be possible to achieve a Class A like behaviour [52] and recover a canard explosion. Of course, the region of small oscillations predicted in the modelling is significantly smaller than the minimum step size of our TLS and so it would be extremely difficult to detect this region. We also believe that it is possible that, depending on device details (such as fabrication methods), the optothermal coupling may vary from device to device and that for sufficiently low values of $c$, a true phase-locked bistability may be possible. In fact, even slightly lower values of $c$ than those analysed here can achieve this.

The novelty of obtaining both Type I and Type II excitability is also noteworthy. To the best of our knowledge, this has not been described for any other optical system in the literature. Thus, the optically injected QD system remains a treasure trove of excitability and related phenomenon and given the intensive ongoing research in neuromorphic dynamics, is of great potential interest to researchers in a range of fields.
[1] B.J. Shastri, A.N. Tait, T. Ferreira de Lima, M.A Nahmias, H.-T. Peng, P.R. Prucnal, Encyclopedia of Complexity and Systems Science, Springer Berlin Heidelberg, R.A. Meyers (ed), 1 (2018)

[2] A. L. Hodgkin and A. F. Huxley, The Journal of physiology 117, 500 (1952)

[3] B. Belousov, Medgiz, Moskow , 145 (1959)

[4] A. M. Zhabotinsky, Biofizika 9, 306 (1964)

[5] F. Plaza, M. Velarde, F. Arecchi, S. Boccaletti, M. Ciofini, and R. Meucci, EPL (Europhysics Letters) 38, 85 (1997)

[6] M. Giudici, C. Green, G. Giacomelli, U. Nespolo, J.R. Tredicce, Phys. Rev. E 55, 6414 (1997)

[7] S. Wieczorek, B. Krauskopf, D. Lenstra, Phys. Rev. Lett. 88, 063901 (2002)

[8] D. Goulding, S.P. Hegarty, O. Rasskazov, S. Melnik, M. Hartnett, G. Greene, J.G. McInerney, D. Rachinskii, G. Huyet, Phys. Rev. Lett. 98, 153903 (2007)

[9] H.J. Wünsche, O. Brox, M. Radziunas, F. Henneberger, Phys. Rev. Lett. 88, 023901 (2001)

[10] A.M. Yacomotti, M.C. Eguia, J. Aliaga, O.E. Martinez, G.B. Mindlin, A. Lipsich, Phys. Rev. Lett. 83, 292 (1999)

[11] B. Kelleher, C. Bonatto, G. Huyet, S.P. Hegarty, Phys. Rev. E 83, 026207 (2011)

[12] B. Garbin, A. Dolcemascolo, F. Prati, J. Javaloyes, G. Tissoni, S. Barland, Physical Review E 95, 012214 (2017)

[13] T. Sorrentino, C. Quintero-Quiroz, A. Aragoneses, M.C. Torrent, C. Masoller, Opt. Exp. 23, 5571 (2015)

[14] G. Giacomelli, M. Giudici, S. Balle, J.R. Tredicce, Phys. Rev. Lett. 84, 3298 (2000)

[15] G.J. Spühler, R. Paschotta, R. Fluck, B. Braun, M. Moser, G. Zhang, E. Gini, U. Keller, J. Opt. Soc. Am. B 16, 376 (1999)

[16] M.A. Nahmias, B.J. Shastri, A.N. Tait, P.R. Prucnal, IEEE J. Sel. Top. Quantum Electron. 19, 1 (2013)

[17] F. Selmi, R. Braive, G. Beaudoin, I. Sagnes, R. Kuszelewicz, S. Barbay, Phys. Rev. Lett. 112, 183902 (2014)

[18] B.J. Shastri, M.A. Nahmias, A.N. Tait, A.W. Rodriguez, B. Wu, P.R. Prucnal, Sci. Rep. 6, 19126 (2016)

[19] S. Barbay, R. Kuszelewicz, A.M. Yacomotti, Opt. Lett. 36, 4476 (2011)

[20] J.L.A. Dubbeldam, B. Krauskopf, D. Lenstra, Phys. Rev. E 60, 6580 (1999)

[21] M.A. Larotonda, A. Hnilo, J.M. Mendez, A.M. Yacomotti, Phys. Rev. A, 65, 033812 (2002)

[22] W. Coomans, L. Gelens, S. Beri, J. Danckaert, G. Van der Sande, Phys. Rev. E, 84, 1 (2011)

[23] T. Van Vaerenbergh, M. Fiers, P. Mechet, T. Spuesens, R. Kumar, G. Morthier, B. Schrauwen, J. Dambre, P. Bienstman, Opt. Exp. 20, 20292 (2012)

[24] A. Hurtado, J. Javaloyes, Appl. Phys. Lett. 107, 241103 (2015)

[25] A. Hurtado, K. Schires, I.D. Henning, M.J. Adams, Appl. Phys. Lett. 100, 103703 (2012)

[26] M. Dillane, D. Goulding, N. Fedorov, I. Dubinkin, T. Erneux, E.A. Viktorov, B. Kelleher, Proc. SPIE 10682 (2018)

[27] B. Kelleher, B. Tykalewicz, D. Goulding, N. Fedorov, I. Dubinkin, T. Erneux, E.A. Viktorov, Sci Rep. 7, 8414 (2017)

[28] M. Brunstein, A.M. Yacomotti, I. Sagnes, F. Raineri, L. Bigot, A. Levenson, Phys. Rev. A 85, 031803 (2012)

[29] A.M. Yacomotti, P. Monnier, F. Raineri, B.B. Bakir, C. Seassal, R. Raj, J.A. Levenson, Phys. Rev. Lett. 97, 143904 (2006)

[30] B. Romeira, J. Javaloyes, C.N. Ironside, J.M.L. Figueiredo, S. Balle, O. Piro, Opt. Exp. 21, 20931 (2013)

[31] B. Romeira, R. Avó, J.L. Figueiredo, S. Barland, J. Javaloyes, Sci. Rep. 6, 19510 (2016)

[32] S. Wieczorek, D. Lenstra, Phys. Rev. E 69, 016218 (2004)

[33] B. Kelleher, D. Goulding, S.P. Hegarty, G. Huyet, D.Y. Cong, A. Martinez, A. Lemaître, A. Ramdane, M. Fischer, F. Gerschütz, J. Koeth, Opt. Lett. 34, 440 (2009)

[34] B. Kelleher, D. Goulding, G. Huyet, E.A. Viktorov, T. Erneux, S.P. Hegarty, Phys. Rev. E 84, 026208 (2011)

[35] M. Turconi, B. Garbin, M. Feyereisen, M. Giudici, S. Barland, Phys. Rev. E 88, 022923B (2013)

[36] B. Garbin, D. Goulding, S.P. Hegarty, G. Huyet, B. Kelleher, S. Barland, Opt. Lett. 39, 1254 (2014)

[37] F. Marino, S. Balle, Phys. Rev. Lett. 94, 094101 (2005)

[38] D. Brunner, M.C. Soriano, C.R. Mirasso, I. Fischer, Nat. Commun. 4, 1364 (2013)

[39] B. Lindner, J. García-Ojalvo, A. Neiman, L. SchimanskyGeier, Phys. Rep. 392, 321-424 (2004)

[40] M. Dillane, B. Tykalewicz, D. Goulding, B. Garbin, S. Barland, and B. Kelleher, Opt. Lett. 44, 347-350 (2019)

[41] T. Erneux and P. Glorieux, Laser Dynamics (Cambridge University Press, Cambridge, 2010)

[42] B. Kelleher, C. Bonatto, P. Skoda, S.P. Hegarty, G. Huyet, Phys. Rev. E 81, 036204 (2010)

[43] S. Barland, O. Piro, M. Giudici, J. R. Tredicce, and S. Balle, Phys. Rev. E 68, 036209 (2003)

[44] Y. A. Rzhanov, H. Richardson, A. Hagberg and J. 
Moloney, Phys. Rev. A 47, 1480 (1993)

[45] A. Tierno, N. Radwell, and T. Ackemann, Phys. Rev. A 84, 043828 (2011)

[46] E. A. Viktorov and T. Erneux, Phys. Rev. E 90, 052914 (2014)

[47] F. Marino, G. Cataln, P. Snchez, S. Balle, and O. Piro, Phys. Rev. Lett. 92, 073901 (2004)

[48] B. Kelleher, D. Goulding, B. Baselga Pascual, S.P. Hegarty, G. Huyet, Phys. Rev. E 85, 046212 (2012)

[49] J. Thévenin, M. Romanelli, M. Vallet, M. Brunel, T. Erneux, Phys. Rev. Lett. 107, 104101 (2011)

[50] B. Kelleher, D. Goulding, B. Baselga-Pascual, S. P.
Hegarty, G. Huyet, Eur. Phys. J. D 58, 175 (2010)

[51] B. Kelleher, D. Goulding, S. P. Hegarty, G. Huyet, E. A. Viktorov, T. Erneux, Optically Injected Single-Mode Quantum Dot Lasers in Quantum Dot Devices (Springer, 2012), Chap. 1, pp. 1-22

[52] B Kelleher, SP Hegarty, G Huyet, Phys. Rev. E 86, 066206 (2012)

[53] J.R. Tredicce, F.T. Arecchi, G.L. Lippi, and G.P. Puccioni, J. Opt. Soc. Am. B 2, 173 (1985)

[54] C. Mayol, R. Toral, C.R. Mirasso and M.A. Natiello, Phys. Rev. A 66, 013808 (2002)

[55] T. Erneux, E.A. Viktorov, B. Kelleher, D. Goulding, S.P. Hegarty, G. Huyet, Opt. Lett. 35, 937 (2010) 